\title{
A family of non-isomorphism results
}

\author{
Collin Bleak · Daniel Lanoue
}

Received: 13 November 2008 / Accepted: 22 September 2009 / Published online: 25 November 2009

(C) The Author(s) 2009. This article is published with open access at Springerlink.com

\begin{abstract}
We calculate the local groups of germs associated with the higher dimensional $\mathrm{R}$. Thompson groups $n V$. For a given $n \in N \cup\{\omega\}$, these groups of germs are free abelian groups of rank $r$, for $r \leq n$ (there are some groups of germs associated with $n V$ with rank precisely $k$ for each index $1 \leq k \leq n$ ). By Rubin's theorem, any conjectured isomorphism between higher dimensional $\mathrm{R}$. Thompson groups induces an isomorphism between associated groups of germs. Thus, if $m \neq n$ the groups $m V$ and $n V$ cannot be isomorphic. This answers a question of Brin.
\end{abstract}

Keywords Higher Dimensional R. Thompson Groups nV · Germs · Rubin's Theorem

Mathematics Subject Classification (2000) 20E32 - 20B22 - 20F65 · 20F38

\section{Introduction}

In the paper [1], Brin introduces a family $n V$ of groups, where $n \in\{1,2, \ldots\} \cup\{\omega\}$. These groups are homeomorphism groups of the Cantor set. However, for each index $n$, it is usual to consider $n V$ as a group of homeomorphisms of $C^{n}$ which is of course homeomorphic to $C$; by definition the elements of $n V$ essentially respect the local product structure on $C^{n}$. Thus, these groups typically are thought of as "higher dimensional" analogues of R. Thompson's group $V=1 \mathrm{~V}$. One current question in the theory of the groups is whether there are indices $m \neq n$ with $m V \cong n V$. In the seminal article [1], Brin shows that the group $2 V$ is not isomorphic to $V$. He also asks in [4] whether $m V$ and $n V$ can be isomorphic if $m \neq n$. In this paper, we answer that question.

C. Bleak $(\otimes)$

Department of Mathematics, University of Nebraska, Lincoln, NE 68588-0130, USA

e-mail: loeksnokes@gmail.com

D. Lanoue

Department of Mathematics, University of California at Berkeley, Berkeley, CA 94720-3841, USA

e-mail: lanoue.d@gmail.com 
While the theory of the groups $n V$ is only in its early development, it is already known that these groups have interesting properties. They are simple (see [2]), for $n \leq \omega$ the group $n V$ is finitely presented (see [5]), and each group $n V$ contains copies of every finite group (since it contains a copy of $V$, which itself has this property). Because of these properties, it is non-trivial to detect whether these groups are pairwise isomorphic through the use of standard algebraic machinery.

Brin shows that $V=1 \mathrm{~V}$ and $2 \mathrm{~V}$ are not isomorphic by taking advantage of dynamical properties of the actions of these groups on the Cantor set. This is enabled by a theorem of Rubin which applies to these groups; any potential pairwise isomorphism would preserve the orbit structure of the actions of corresponding group elements on the Cantor set. To prove contradiction, Brin finds an element in $2 \mathrm{~V}$ which acts with non-trivial entropy and shows that no element of $V$ acts in such a fashion.

The main difficulty in proving the generalized non-isomorphism result lay in finding the appropriate invariant of topological conjugacy to distinguish the groups in the family $n V$. While coming to understand that the groups in $n V$ do not appear to be generally separable by calculations of topological entropy, the authors gathered the appropriate information for the calculation of the local groups of germs. The second author then discovered that the local groups of germs actually captured precisely the information we needed to separate the groups in $n V$.

Given an index $n$ and a point $x \in C^{n}$, we calculate the local group of germs $G_{(n V, x)}$ of $n V$ at $x$. We obtain the following result.

Theorem 1 Let $n \in\{1,2, \ldots\} \cup\{\omega\}$ and $x \in C^{n}$. Suppose that $|x|$ is the number of coordinates of $x$ which are rational. The group of germs $G_{(n V, x)}$ of $n V$ at $x$ is isomorphic to $Z^{|x|}$, the free abelian group of rank $|x|$.

Rubin's theorem now applies so we obtain the generalized non-isomorphism result.

Theorem 2 Let $m, n \in\{1,2, \ldots\} \cup\{\omega\}$, with $m \neq n$, then $m V$ is not isomorphic to $n V$.

\section{Background requirements}

Throughout this section, we will provide the background and set the stage for that proof.

\subsection{Rubin's theorem}

Brin indicates in [1] that the groups $V$ and $2 V$ act on $C$ and $C^{2}$ in such a way that Rubin's Theorem [6] applies; any isomorphism $\phi: V \rightarrow 2 V$ induces a unique homeomorphism $\psi: C \rightarrow C^{2}$, so that for $v \in V, \phi(v)=\psi \circ v \circ \psi^{-1}$. Brin does not prove this fact fully, but gives the reader sufficient explanation to verify the result for themselves.

Brin's argument in [1] does not directly demonstrate that Rubin's Theorem applies to any possible isomorphism between $m V$ and $n V$ with $m$ or $n \geq 3$. However, with a small amount of work Brin's argument can be generalized to show that Rubin's theorem still applies. We will assume below that the reader has seen that this is the case.

\subsection{A group of germs}

As mentioned, our key invariant will be the group of germs at a fixed point, for which we now recall the definition. Suppose $X$ is a topological space, and $H$ is a subgroup of the full group of homeomorphisms of $X$. For any $x \in X$, define the set $\operatorname{Fix}_{(H, x)}=\{h \in H \mid h(x)=x\}$. 
We place an equivalence relation on $\operatorname{Fix}_{(H, x)}$. We say that $f, g \in \operatorname{Fix}_{(H, x)}$ are equivalent if there is a neighborhood $U$ of $x$ so that $\left.f\right|_{U}=\left.g\right|_{U}$. In this case we write $f \sim_{x} g$ and we denote the equivalence class (the germ) of any element $h \in \operatorname{Fix}_{(H, x)}$ by $[h]_{x}$. We further denote the set of germs that result by $G_{(H, x)}$.

The following is standard. It is also given explicitly as a portion of Lemma 3.4 in [3].

Lemma 1 Given a topological space $X$, a point $x \in X$, and a group of homeomorphisms $H$ of $X$, the set $G_{(H, x)}$ forms a group under the operation $[f]_{x} *[g]_{x}=[f g]_{x}$.

$2.3 C^{n}, n$-rectangles, and the groups $n V$

There is a well-known correspondence between finite binary strings, and subsets of the Cantor set $C$. We interpret a string as inductive choices of halves. For instance, the string "011" means take the left half of the Cantor set (throwing out the right half), then take the right half of what remains, and finally pass to the right half of that. The diagram below illustrates this notation.

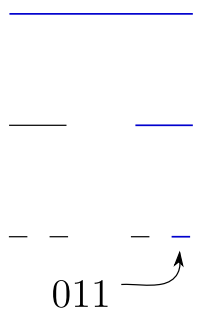

If we pass to the limit and consider infinite binary strings, we obtain the standard bijection from $2^{N}$, the space of all infinite strings in the alphabet $\{0,1\}$, to the Cantor set. That is, we abuse notation by having an element $s$ of the Cantor set correspond to a map $s: N \rightarrow\{0,1\}$. In this notation, we would denote $s(0)$ by $s_{0}$, so that we can write $s$ as an infinite string $s=s_{0} s_{1} \ldots$. In this case, we can define a prefix of $s$ of length $r$ as the finite substring $s_{0} \cdots s_{r-1}$. We will call an infinite binary string $w$ an infinite tail of $s$ if $s=P w$ for some finite prefix $P$ of $s$.

Elements $s, t \in 2^{N}$ are near to each other when they have long common prefixes. This induces the standard topology of the Cantor set. In this description, a point $z$ of the Cantor set will be rational if and only if it corresponds to an infinite string of the form $P \bar{w}=P w w \ldots$, where $P$ is a prefix string and $w$ is some non-empty finite string.

We will now fix $n$ as a positive integer. Let us first define a special class of subsets of $C^{n}$. $R$ is an $n$-rectangle in $C^{n}$ if there is a collection of finite binary strings $P_{0}, P_{1}, \ldots, P_{n-1}$ so that

$$
R=\left\{z \in C^{n} \mid z=\left(x_{0}, x_{1}, x_{2}, \ldots, x_{n-1}\right) \text { and } x_{i}=P_{i} z_{i} \text { where } z_{i} \in 2^{N}\right\} .
$$

In this case, by an abuse of notation, we will say $R=\left(P_{0}, P_{1}, \ldots, P_{n-1}\right)$.

We now define a special class of maps, $n$-rectangle maps, as follows. Suppose $D=$ $\left(P_{0}, P_{1}, \ldots, P_{n-1}\right)$ and $R=\left(Q_{0}, Q_{1}, \ldots, Q_{n-1}\right)$ are $n$-rectangles. We define the $n$-rectangle map $\tau_{(D, R)}: D \rightarrow R$ by the rule $z \mapsto z^{\prime}$ where the $i$ 'th coordinate $P_{i} z_{i}$ of $z$ determines the $i$ 'th coordinate $Q_{i} z_{i}$ of $z^{\prime}$. We will also refer to these as prefix maps.

We note that a prefix map $\tau_{(D, R)}$ (as above) scales dimension $i$ in accord with the length of $P_{i}$ and the length of $Q_{i}$. For instance, if $P_{i}=1$ and $Q_{i}=011$, then in dimension $i$, the map will take a half of the Cantor set and map it affinely over an eighth of the Cantor set. 
In particular, any such map will have a scaling factor of $2^{n}$ for $n \in Z$. (In terms of metric scaling, viewing the Cantor set as the standard deleted-middle-thirds subset of the interval, the scaling factors would of course be $3^{n}$. We will use the $2^{n}$ point of view in the remainder of this note.)

We can now define a pattern to be a partition of $C^{n}$ into a finite collection of pairwise disjoint $n$-rectangles. An element $f$ of $n V$ now corresponds to a homeomorphism from $C^{n}$ to $C^{n}$ for which there is an integer $k$, a domain pattern $D$, and a range pattern $R$, each pattern with $k$ rectangles, so that $f$ can be realized as the union of $k$ disjoint $n$-rectangle maps, each carrying an $n$-rectangle of $D$ to an $n$-rectangle of $R$. It should be noted that two different pairs of partitions can correspond to the same map. The diagram below demonstrates a typical such map in $2 V$.

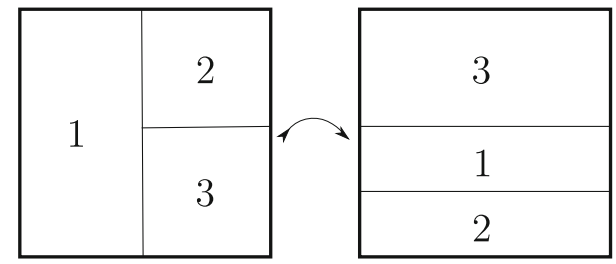

In the case of $\omega V$, the $\omega$-rectangles are only allowed to restrict the domain in finitely many dimensions. That is, $R=\left(P_{0}, P_{1}, P_{2}, \ldots\right)$ is an $\omega$ rectangle if and only if only finitely many of the $P_{i}$ are non-empty strings. Thus, $\omega V$ can be thought of as a direct union of the $n V$ groups for finite $n$.

\subsection{Rubin's Theorem and some groups of germs}

In order to state Theorem 3 below, we need to give a further definition. If $X$ is a topological space and $F$ is a subgroup of the group of homeomorphisms of $X$, then we will say that $F$ is locally dense if and only if for any $x \in X$ and open neighborhood $U$ of $x$ the set

$$
\left\{f(x)|f \in F, f|_{(X-U)}=\left.1\right|_{(X-U)}\right\}
$$

has closure containing an open set.

The following is the statement of Rubin's Theorem, as given by Brin as Theorem 2 in [1]. It is a modification of Rubin's statement Theorem 3.1 in [6], which statement appears to contain a minor technical error.

Theorem 3 (Rubin) Let $X$ and $Y$ be locally compact, Hausdorff topological spaces without isolated points, let $H(X)$ and $H(Y)$ be the automorphism groups of $X$ and $Y$, respectively, and let $G \subseteq H(X)$ and $H \subseteq H(Y)$ be subgroups. If $G$ and $H$ are isomorphic and are both locally dense, then for each isomorphism $\phi: G \rightarrow H$ there is a unique homeomorphism $\psi: X \rightarrow Y$ so that for each $g \in G$, we have $\phi(g)=\psi g \psi^{-1}$.

If we combine Rubin's Theorem with our previous work on the group of germs, we get a lemma which appears to be a very mild extension of Lemma 3.4 from [3].

Lemma 2 Suppose $X$ and $Y$ are locally compact, Hausdorff topological spaces without isolated points, and that $G$ and $H$ are respectively subgroups of the homeomorphism groups of $X$ and $Y$, so that $G$ and $H$ are both locally dense. Suppose further that $\phi: G \rightarrow H$ is an isomorphism, and that $\psi$ is the homeomorphism induced by Rubin's Theorem. If $x \in X$ and $y \in Y$ so that $\psi(x)=y$, then $\psi$ induces an isomorphism $\bar{\psi}: G_{(G, x)} \rightarrow G_{(H, y)}$. 
Proof This is a straightforward exercise in calculation.

For $[v]_{x} \in G_{(G, x)}$, define $\bar{\psi}\left([v]_{x}\right)=\left[\psi v \psi^{-1}\right]_{y}$.

We first show that $\bar{\psi}$ is well defined. Let $f \sim_{x} g$ with $f, g \in F i x_{(G, x)}$, so that $\left.f\right|_{U}=\left.g\right|_{U}$ with $U$ an open neighborhood of $x$. Then $N=\psi(U)$ is an open neighborhood of $y=\psi(x)$ since $\psi$ is a homeomorphism. For $z \in N, \psi^{-1}(z) \in U$, so $\psi f \psi^{-1}(z)=\psi g \psi^{-1}(z)$. Thus $\left[\psi f \psi^{-1}\right]_{y}=\left[\psi g \psi^{-1}\right]_{y}$.

Now we show that $\bar{\psi}$ is a homomorphism. We have

$$
\begin{aligned}
\bar{\psi}\left([f]_{x}[g]_{x}\right) & =\bar{\psi}\left([f g]_{x}\right)=\left[\psi f g \psi^{-1}\right]_{y}=\left[\psi f \psi^{-1} \psi g \psi^{-1}\right]_{y} \\
& =\left[\psi f \psi^{-1}\right]_{y}\left[\psi g \psi^{-1}\right]_{y}=\bar{\psi}\left([f]_{x}\right) \bar{\psi}\left([g]_{x}\right) .
\end{aligned}
$$

Finally, we show that $\bar{\psi}$ is a bijection. Since $\psi^{-1}$ conjugates $H$ to $G$, we also have an induced map $\overline{\psi^{-1}}$. Now, direct calculation as above shows that $\overline{\psi^{-1}} \circ \bar{\psi}$ and $\bar{\psi} \circ \overline{\psi^{-1}}$ are the identity maps on $G_{(G, x)}$ and $G_{(H, y)}$ respectively.

\section{Conclusion}

Below, we give our proofs using notation for $m<n<\omega$. The result for $n=\omega$ is immediate from the arguments below when one considers $\omega V$ as a direct limit of the $n V$ in the natural fashion.

We now prove Theorem 1. Namely, the group of germs of $n V$ at $x \in C^{n}$ is isomorphic with the free abelian group with rank equal to the number of rational coordinates of $x$.

Proof Assume $x=\left(x_{1}, \ldots, x_{n}\right) \in C^{n}$ and let $|x|=k$ denote the cardinality of the set $\left\{i: x_{i}\right.$ rational $\}$. Assume without meaningful loss of generality that $x_{0}, \ldots, x_{k-1}$ are the rational coordinates. Let us write $x_{i}=A_{i} \overline{w_{i}}$ for each index $i$ with $0 \leq i \leq k-1$, where $A_{i}$ is the shortest prefix so that $x_{i}$ can be written in this fashion, and where $w_{i}$ is the shortest word so that an infinite tail of $x_{i}$ is of the form $\overline{w_{i}}$. Suppose $f \in n V$ with $f(x)=x$, and let $j$ be the minimal integer so that $f$ admits a decomposition as a union of $j$ disjoint $n$-rectangle maps, $\left\{f_{i}: D_{i} \rightarrow R_{i} \mid 1 \leq i \leq j\right\}$, with $f=f_{1} \cup f_{2} \ldots \cup f_{j}$.

Assume further that $a$ is the index so that $x \in D_{a}$. By our earlier assumptions, $x \in R_{a}$ as well. We can now restrict our attention to $\tau_{\left(D_{a}, R_{a}\right)}$. By definition there are finite strings $P_{(a, i)}$ and $Q_{(a, i)}$ so that $R_{a}=\left(P_{(a, 0)}, P_{(a, 1)}, \ldots, P_{(a, n-1)}\right)$ and $D_{a}=\left(Q_{(a, 0)}\right.$, $\left.Q_{(a, 1)}, \ldots, Q_{(a, n-1)}\right)$.

Since $x$ is fixed by $f$, we must have that for each index $m \geq k, P_{(a, m)}=Q_{(a, m)}$. Also, we see that for each index $m<k, P_{(a, m)}=A_{m}\left(w_{m}\right)^{s_{m}} c_{m}$ and $Q_{(a, m)}=A_{m}\left(w_{m}\right)^{t_{m}} c_{m}$ for some non-negative integers $s_{m}$ and $t_{m}$, and some prefix $c_{m}$ of $w_{m}$. Define for $f$, the tuple $\left(s_{0}-t_{0}, s_{1}-t_{1}, \ldots, s_{k-1}-t_{k-1}\right) \in Z^{k}$. We will call this association $\hat{\sigma}_{x}:$ Fix $x_{(n V, x)} \rightarrow Z^{k}$.

If $g, h \in F i x_{(n V, x)}$, with $[g]_{x}=[h]_{x}$, then $\hat{\sigma}_{x}(g)=\hat{\sigma}_{x}(h)$, as otherwise the scaling of the two maps in some dimension could not be the same in some small neighborhood of $x$.

The map $\hat{\sigma}_{x}$ induces a set map $\sigma_{x}: G_{(n V, x)} \rightarrow Z^{k}$. The reader may now confirm that the map $\sigma_{x}$ is in fact an isomorphism of groups.

We now prove Theorem 2, that if $m \neq n$ then $m V \approx n V$.

Proof Suppose $m$ and $n$ are valid indices, with $m<n$, and suppose $\phi: n V \rightarrow m V$ is an isomorphism, with $\psi: C^{n} \rightarrow C^{m}$ the homeomorphism induced by Rubin's Theorem. Let $x$ be a point in $C^{n}$ with all $n$ coordinates rational, and let $y=\psi(x)$. The map $\psi$ induces an isomorphism $\bar{\psi}: G_{(n V, x)} \rightarrow G_{(m V, y)}$. But observe, the rank of $G_{(n V, x)}$ is $n$, while the rank of $G_{(m V, y)}$ must be less than or equal to $m$. Thus, no such isomorphism $\bar{\psi}$ can exist. 
Open Access This article is distributed under the terms of the Creative Commons Attribution Noncommercial License which permits any noncommercial use, distribution, and reproduction in any medium, provided the original author(s) and source are credited.

\section{References}

1. Brin, M.: Higher dimensional Thompson groups. Geom. Dedicata 108, 163-192 (2004)

2. Brin, M.: On the baker's map and the simplicity of the generalized Thompson groups $n V$. Publ. Mat. (to appear)

3. Brin, M.: The chameleon groups of Richard J. Thompson: automorphisms and dynamics. Inst. Hautes Tudes Sci. Publ. Math. 84, 5-33 (1996)

4. Brin, M.: Cousins of Thompson's groups, III (Higher dimensional groups). Thompson's Groups: New Developments and Interfaces, CIRM, Marseille, France (2008)

5. Hennig, J., Matucci, F.: Presentations for the groups $n V$ (in preparation)

6. Rubin, M.: Locally Moving Groups and Reconstruction Problems, Ordered Groups and Infinite Permutation Groups. pp. 121-157. Kluwer Academic Publisher, Dordrecht (1996) 